\title{
ANESTESIA GERAL COM PROPOFOL EM CÃES PRÉ-TRATADOS COM ACEPROMAZINA E ALFENTANIL
}

\author{
GENERAL ANESTHESIA WITH PROPOFOL IN DOGS PREMEDICATED WITH \\ ACEPROMAZINE AND ALFENTANIL
}

\author{
Silvia Renata Gaido Cortopassi ${ }^{1}$ Marlene Pezzutti Holzchuh ${ }^{2}$ \\ Denise Tabacchi Fantoni ${ }^{1}$
}

RESUMO

Foram utilizados 30 cães, sem raça definida, adultos, (10 machos e 20 fêmeas), com peso médio de $14,44 \pm$ $2,72 \mathrm{~kg}$, distribuídos em três grupos. O grupo I (GI) recebeu a associação de $0,1 \mathrm{mg} / \mathrm{kg}$ de acepromazina, 0,05mg/kg de alfentanil e 6,0mg/kg de propofol; o grupo $H$ (GII) $0,1 \mathrm{mg} / \mathrm{kg}$ de acepromazina, $O, 1 \mathrm{ml} / \mathrm{kg}$ de solução fisiológica e $6,0 \mathrm{mg} / \mathrm{kg}$ de propofol; e o grupo III (GIII) recebeu $0,05 \mathrm{ml} / \mathrm{kg}$ de solução fisiológica, $0,05 \mathrm{mg} / \mathrm{kg}$ de alfentanil e $6,0 \mathrm{mg} / \mathrm{kg}$ de propofol. Todos os fármacos foram administrados através da via intravenosa. Houve bradicardia intensa e de curta duração nos animais dos grupos I e III após a administração do alfentanil, tendo a frequência cardíaca se restabelecido após a indução da anestesia. No atinente à pressão arterial, os animais dos grupos $l$ e II apresentaram hipotensão acentuada, havendo recuperação dos índices pressóricos apenas do GII. As três associações anestésicas promoveram alterações dos valores obtidos na hemogasometria arterial, verificando-se aumento da $\mathrm{PaCO}_{2}$ e decréscimo do $\mathrm{pH}, \mathrm{PaO}_{2}$ e $\mathrm{SaO}_{2}$ o que se traduz em depressão respiratória, sendo que nos animais tratados com alfentanil o grau de depressão foi mais grave. $O$ alfentanil ainda promoveu aumento significativo da frequência respiratória. A indução anestésica nos três grupos experimentais foi suave, não se observando fenômenos excitatórios. O propofol, como agente indutor, mostrou ser fármaco potente e seguro, não tendo ocorrido depressão das funções cardiovasculares e respiratórias. A acepromazina e a associação de acepromazina e alfentanil como medicações pré-anestésicas são efetivas, pois contribuíram para obtenção de técnica anestésica isenta de efeitos adversos, não se observando excitação ou mioclonia.

Palavras-chave: propofol, acepromazina, alfentanil, cães, anestesia.

\section{SUMMARY}

The objective of this study was to evaluate the effects of three different associations: acepromazine $0,1 \mathrm{mg} / \mathrm{kg}$, alfentanil 0,05mg/kg and propofol 6,0mg/kg (Group l); acepromazine $0,1 \mathrm{mg} / \mathrm{kg}, 0,9 \% \mathrm{NaCI} 0,05 \mathrm{ml} / \mathrm{kg}$ and propofol 6,0mg/kg (Group II); $0,9 \% \mathrm{NaCI} 0,1 \mathrm{m \ell} \mathrm{kg}$, alfentanil $0,05 \mathrm{mg} / \mathrm{kg}$ and propofol $6,0 \mathrm{mg} / \mathrm{kg}$ (Group III). Thirty healthy adult mongrel dogs (10 males and 20 females; mean weight, 14,44 $\pm 2,72 \mathrm{~kg}$ ) were divided in three groups randomiy assigned to one of the associations. Short-term bradicardia was observed in groups I and III immediately after the administration of alfentanil. The heart rate retumed to control values at the end of the evaluation period. $A U$ the anesthetic procedures caused severe alterations in respiratory function with a significant decrease in $\mathrm{PaO}_{2}, p \mathrm{H}$ and $\mathrm{SaO}_{2}$ and rise of $\mathrm{PaCO}_{2}$. Respiratory depression was more intense when alfentanil was used. Respiratory rate with this drug increased significantiy. In the acepromazine-propofol group, the some alterations could be noticed but not so severe. The induction of anesthesia in all groups was uneventful and excitement free and the intubation was easily performed. Anesthesia recovery was smooth, fast and excitment free. These observations confirm that propofol is in fact an effective and safe induction agent once we did not observe life-threatining cardiovascular depression and undesirable side effects. Both acepromazine alone and acepromazine pius alfentanil are effective premedication agents once they contributed to an uneventful induction and recovery of anesthesia without excitatory fenômeno or myoclonus, with prolongation of the anesthetic time of propofol.

Key words: propofol, acepromazine. alfentanil, dogs, anesthesia.

\section{INTRODUÇÃO}

O propofol (2,6 di-isopropylphenol) é agente hipnótico intravenoso, disponível como emulsão de óleo em água, contendo óleo de soja, lecitina, ovo e glicerol e não contém preservativos. Tem pH neutro, é isotônico e sua formulação apenas é efetiva quando administrada através da via intra-

\footnotetext{
${ }^{1}$ Médico Veterinário, Professor, Doutor, Faculdade de Medicina Veterinária e Zootecnia (FMVZ), Universidade de São Paulo (USP). Endereço para correspondência: Av.: Angélica, 765/902, 01227-000, São Paulo - SP. E-mail: silcorto@nw.com.br.

${ }^{2}$ Médico Veterinário, Professor, Doutor, Aposentado, FMVZ, USP. 
venosa. Tem sido utilizado em dose única ou na manutenção da anestesia através da administração contínua (HALL \& CHAMBERS, 1987; WATKINS et al., 1987; CHAMBERS, 1988; GENEVOIS et al., 1988; FAU et al., 1989; MORGAN \& LEGGE, 1989; WEAVER \& RAPTOPOULOS, 1990; GEEL, 1991; ROBERTSON et al., 1991; VAINIO, 1991; WATNEY \& PABLO, 1992). A anestesia promovida pelo propofol em cães é caracterizada por rápido início, curta duração e suave recuperação (MORGAN \& LEGGE, 1989; SEBEL \& LOWDON, 1989; ILKIW et al., 1992). Não tem efeito cumulativo após aplicações repetidas. Várias pesquisas têm mostrado que o propofol promove algumas alterações cardiorrespiratórias em cães prétratados ou não com tranquilizantes, sedativos ou outros agentes (WATKINS et al., 1987; MORGAN \& LEGGE, 1989; WEAVER \& RAPTOPOULOS, 1990; VAINIO, 1991; SMITH et al., 1993). As medicações pré-anestésicas podem promover alterações no sistema nervoso central (SNC), cardiovascular, respiratório assim como nos processos metabólicos. Esses efeitos podem relacionar-se com mudanças na frequência cardíaca ou pressão arterial, e em maior depressão do SNC fazendo com que a resposta anestésica do propofol seja de maior duração, por exemplo. Dessa forma, objetivou-se, com este estudo, avaliar os efeitos cardiorrespiratórios, a qualidade de indução e recuperação do agente intravenoso propofol antecedido do uso de três diferentes pré-medicações, nas quais se utilizou o tranquilizante acepromazina e o opióide alfentanil, associados e cada um isoladamente.

\section{MATERIAL E MÉTODOS}

Foram utilizados 30 cães adultos, 10 machos e 20 fêmeas, sem raça definida, pesando entre 10 a $19 \mathrm{~kg}$, provenientes do Canil Geral da Faculdade de Medicina Veterinária e Zootecnia da Universidade de São Paulo. Os animais foram submetidos a exame clínico completo, e submetidos a jejum alimentar prévio de 12 horas e hídrico de quatro horas antes dos procedimentos anestésicos. Os animais foram distribuídos aleatoriamente em três grupos de 10 animais cada, os quais foram organizados da seguinte forma: Grupo I (GI) - os animais foram pré-tratados com $0,1 \mathrm{mg} / \mathrm{kg}$ de acepromazina; decorridos seis minutos, receberam alfentanil na dose de $0,05 \mathrm{mg} / \mathrm{kg}$, após quatro minutos, a indução da anestesia foi realizada com $6,0 \mathrm{mg} / \mathrm{kg}$ de propofol durante o período de 20 segundos; Grupo II (GII) - os animais receberam a mesma dose de acepromazina ,e decorridos seis minutos, foi administrada solução fisiológica de cloreto de sódio a $0,9 \%$ no volume de $0,1 \mathrm{~m} \ell / \mathrm{kg}$, correspondente ao mesmo volume de alfentanil empregado nos demais grupos; posteriormente, a anestesia foi induzida com $6,0 \mathrm{mg} / \mathrm{kg}$ de propofol durante o período de 20 segundos; Grupo III (GIII) os animais foram pré-tratados com $0,05 \mathrm{ml} / \mathrm{kg}$ de solução fisiológica de cloreto de sódio a $0,9 \%$ correspondente ao mesmo volume de acepromazina empregado nos demais grupos; decorridos seis minutos, aplicou-se alfentanil na dose de $0,05 \mathrm{mg} / \mathrm{kg}$, e após quatro minutos, os cães receberam $6,0 \mathrm{mg} / \mathrm{kg}$ de propofol durante o período de 20 segundos. Todos os fármacos foram administrados através da via intravenosa. Foram avaliados os seguintes parâmetros: frequência (FC) e ritmo cardíaco com auxílio do eletrocardiógrafo, na velocidade de $25 \mathrm{~mm} / \mathrm{sec}$, resultando da divisão do número absoluto 1500 pelo intervalo R-R em milissegundos, tendo sido realizadas as derivações do plano frontal (bipolares DI,II,III e unipolares: aVR, aVL e aVF)(os animais foram mantidos em decúbito lateral direito e os eletrodos fixados no membro torácico abaixo do olécrano e no membro pélvico sobre os ligamentos patelares); as pressões sistólica (PÁS), média (PAM) e diastólica (PAD) foram avaliadas através de monitor não invasivo onde o manguito foi colocado no terço proximal do rádio (SAWYER $\boldsymbol{e t}$ al., 1991), obtendo-se, dessa maneira, a pressão na artéria braquial; a frequência respiratória (FR) através da inspeção dos movimentos da parede torácica no decorrer de um minuto; análise do $\mathrm{pH}$, pressão parcial arterial de oxigênio e dióxido de carbono $\left(\mathrm{PaO}_{2}\right.$ e $\left.\mathrm{PaCO}_{2}\right)$, bicarbonato plasmático $\left(\mathrm{HCO}_{3}{ }^{-}\right)$e saturação da oxi-hemoglobina arterial $\left(\mathrm{SaO}_{2}\right)$ através da coleta de sangue da artéria femoral submetido ao analisador de $\mathrm{pH}$ e gases sanguíneos. A coleta de sangue para hemogasometria foi realizada através de catéter de calibre 22 fixado na artéria femoral. O volume da amostra foi de $0,5 \mathrm{~m} \ell$ em seringa descartável contendo $0,1 \mathrm{~m} \ell$ de heparina (TERZI, 1992), sendo a agulha vedada por tampa de borracha, evitando assim contato do sangue com o ar ambiente. Em seguida, a amostra foi acondicionada em recipiente isotérmico contendo gelo e água, para posterior análise em analisador de $\mathrm{pH}$ e gases sangüíneos. Todas as amostras foram analisadas ao término do experimento, com intervalo máximo de três horas entre coleta e análise.

As avaliações dos parâmetros vitais (FC e ritmo, FR, PÁS, PAM, PAD) foram realizadas: imediatamente antes da aplicação da acepromazina ou da solução fisiológica de cloreto de sódio a $0,9 \%$ (GIII); após três minutos da administração do alfentanil ou da solução fisiológica de cloreto de 
sódio a $0,9 \%$ (GII), e aos um, cinco, 10, 20, 30 e 60 minutos após a administração do propofol. A hemogasometria arterial foi realizada imediatamente antes da administração da acepromazina e da solução fisiológica de cloreto de sódio a $0,9 \%$, três minutos após a aplicação do alfentanil ou da solução fisiológica de cloreto de sódio a $0,9 \%$, e cinco, 20 e 60 minutos após a administração do propofol. A avaliação anestesiológica foi realizada através da verificação dos períodos de latência e hábil do propofol, e através da observação da qualidade da indução anestésica avaliada pêlos seguintes itens: facilidade da intubação orotraqueal e presença (+) ou ausência (-) de excitação, através de movimentos de pedalagem e vocalização. A analgesia foi avaliada através de estímulo doloroso induzido por pinçamento da pele e presença ou não de reflexo interdigital nos membros posteriores. A resposta a esses estímulos foi graduada segundo o escore: (3) excelente: ausência de reação do paciente; (2) - boa: raras reações não significativas; (1) - razoável: reações mais pronunciadas e (0) - ruim: impossibilidade de qualquer estimulação. $\mathrm{O}$ relaxamento muscular foi avaliado segundo o escore proposto por FAU et al. (1989): (3) - intenso: flacidez muscular total; (2) - regular: discreto tônus muscular; (1) - leve: importante tônus muscular e (0) - ausente: contrações durante manipulações. As avaliações da analgesia e do relaxamento muscular foram realizadas apenas após a administração do propofol (um, cinco, 10, 20, 30 e 60 minutos). A qualidade da recuperação anestésica foi avaliada pela presença ou ausência de ataxia e excitação.

Os resultados obtidos foram confrontados estatisticamente através de provas paramétricas, tendo-se utilizado para tal a análise de variância (ANOVA), seguida do teste de Bonferroni (Instat Graphpad Software) para a comparação dos diferentes tempos de observação de um mesmo grupo. Para a análise entre os tempos dos três grupos experimentais, utilizou-se a ANOVA, seguida do teste de Schieffe (Prostat). O grau de significância estabelecido foi de $5 \%(\mathrm{p}<0,05)$. Os escores obtidos na avaliação da analgesia e do relaxamento muscular foram avaliados estatisticamente através do teste de Friedman para medidas repetidas não paramétricas.

\section{RESULTADOS}

No atinente a FC, todos os grupos apresentaram redução nos valores após a administração do agente pré-anestésico, sendo essa diminuição significativa nos animais que receberam alfentanil; posterior à aplicação do propofol, esses valores tenderam a aumentar, mantendo-se constantes, porém ainda inferiores nos animais do GIII (Tabela 1).
Em relação ao ritmo cardíaco, foram observadas arritmias em $8,7 \%$ dos momentos analisados do grupo I, sendo que $100 \%$ desses eventos foram bloqueio átrio-ventricular (BAV) de $2^{\circ}$ grau, Mobitz tipo I. Cerca de 42,8\% dos BAV ocorreram aos três minutos da administração do alfentanil e 14,3\% antes da aplicação dos agentes pré-anestésicos. Verificou-se a presença de ritmo sinusal em $100 \%$ dos animais por período de 20 minutos após a administração do propofol. Já nos animais do grupo II, observou-se aparecimento de BAV de $2^{\circ}$ grau, Mobitz tipo I em 8,7\% dos momentos analisados. Cerca de $85,7 \%$ desses distúrbios ocorreram após a administração da medicação pré-anestésica. Houve presença de ritmo sinusal apenas um minuto após a administração do propofol. Em 15\% dos momentos analisados do grupo III, verificou-se presença de distúrbios da condução (BAV e ritmo juncional). Cerca de 58,3\% desses distúrbios ocorreram aos três minutos da administração do alfentanil e $16,6 \%$ previamente a ele. Dos distúrbios observados, $83,3 \%$ foram BSA de $2^{\circ}$ grau tipo I e o restante ritmos juncionais. Em relação à PÁS, os valores obtidos previamente à aplicação dos fármacos nos animais dos grupos I e II foram superiores aos encontrados nos demais momentos de avaliação. No que diz respeito a análise comparativa entre os grupos, apenas os animais do GIII apresentaram valores significativamente superiores quando comparados aos demais grupos $(\mathrm{p}<0,05)$. Os valores da PAM reduziram significativamente no decorrer do experimento quando compararam-se os valores controle com aqueles obtidos após a administração dos fármacos nos três grupos experimentais. $\mathrm{Na}$ análise comparativa, os animais do GIII apresentaram valores superiores após a administração da MPA e aos cinco e 10 minutos pós-propofol, em relação ao GI, tendo sido a diferença estatisticamente significativa. Aos 30 minutos pós-propofol, o GIII mostrou valores superiores e estatisticamente significativos em relação aos dois grupos. Os valores controle da PAD do GI mostraram-se superiores em relação aos demais momentos de observação $(\mathrm{p}<0,05)$; no GII, os valores obtidos aos cinco, 10, 20 e 30 minutos pós-propofol mostraram-se significativamente inferiores quando comparados com os valores controle. Aos 10 minutos pós-propofol, houve diminuição estatisticamente significativa em relação aos níveis controle nos animais do GIII. O grupo III apresentou valores superiores em relação ao grupo I após a administração da medicação pré-anestésica, e aos 10 minutos pós-propofol $(\mathrm{p}<0,05)$. Decorridos 20 e 60 minutos da administração do propofol, o GIII mostrou valores superiores em relação aos demais grupos $(\mathrm{p}<0,05)$ (Tabela 1$)$. Houve aumento significativo na FR aos 10 minutos pós-propofol 
Tabela 1 - Valores médios e desvios-padrões da frequiência cardíaca (FC) (batimentos/minuto), pressão arterial sistólica (PAS), média (PAM) e distólica (PAD) ( $\mathrm{mmHg}$ ), freqüência respiratória (FR) (movimentos respiratórios/minuto), pH arterial, pressão parcial de oxigênio ( $\mathrm{PaO} 2)$ e dióxido de carbono $\left(\mathrm{PaCO}_{2}\right)$ no sangue arterial (mmHg) de cães tratados com acepromazina, alfentanil e propofol (Grupo I), acepromazina e propofol (Grupo II) e alfentanil e propofol (Grupo III) nos diversos tempos de avaliação.

\begin{tabular}{|c|c|c|c|c|c|c|c|c|c|}
\hline \multirow[b]{2}{*}{ Parâmetros } & \multirow[b]{2}{*}{ Grupos } & \multirow[b]{2}{*}{ Valores Controle } & \multirow[b]{2}{*}{ Pós-MPA } & \multicolumn{6}{|c|}{ Minutos após Propofol } \\
\hline & & & & 1 & 5 & 10 & 20 & 30 & 60 \\
\hline \multirow{3}{*}{$\begin{array}{c}\text { FC } \\
(\mathrm{bat} / \mathrm{min})\end{array}$} & $\mathbf{I}$ & $113,5 \pm 24,9^{a}$ & $71,4 \pm 17,2^{\mathrm{a}}$ & $118,6 \pm 26,8^{a}$ & $109,8 \pm 25,9^{\mathrm{a}}$ & $105,2 \pm 21,4^{\mathrm{a}}$ & $120,3 \pm 23,8^{\mathrm{a}}$ & $121,6 \pm 30,5^{\mathrm{a}}$ & $124,1 \pm 35,5^{\mathrm{a}}$ \\
\hline & II & $105,7 \pm 14,4^{a}$ & $94,3 \pm 15,0^{1}$ & $116,0 \pm 18,1^{a, b}$ & $103,2 \pm 14,8^{a}$ & $98,3 \pm 11,5^{\text {a, }}$ & $91,3 \pm 10,3^{b}$ & $93,9 \pm 11,6^{b}$ & $102,2 \pm 18,4^{\text {a.b }}$ \\
\hline & III & $95,7 \pm 8,0^{a}$ & $57,3 \pm 12,7^{z}$ & $93,8 \pm 13,2^{b}$ & $91,3 \pm 14,1^{\text {a }}$ & $81,5 \pm 12,6^{b}$ & $78,6 \pm 12,5^{b . c}$ & $78,5 \pm 14,8^{\mathrm{b} . c}$ & $88,3 \pm 14,4^{b}$ \\
\hline \multirow{3}{*}{$\begin{array}{c}\text { PAS } \\
(\mathrm{mmHg})\end{array}$} & I & $137,8 \pm 5,75^{\mathrm{a}}$ & $118,3 \pm 11,7^{a}$ & $112,9 \pm 13,5^{\mathrm{a}}$ & $107,5 \pm 14,0^{\mathrm{a}}$ & $105,2 \pm 8,2^{\text {a }}$ & $113,9 \pm 11,3^{a}$ & $116,1 \pm 11,3^{a}$ & $117,9 \pm 9,8^{a}$ \\
\hline & II & $129,7 \pm 7,7^{b}$ & $122,1 \pm 14,5^{a}$ & $115,6 \pm 19,1^{a . b}$ & $112,3 \pm 17,2^{\mathrm{a}}$ & $114,7 \pm 8,7^{a}$ & $114,7 \pm 10,7^{a}$ & $117,0 \pm 14,6^{\mathrm{a}}$ & $116,9 \pm 12,5^{2}$ \\
\hline & III & $132,5 \pm 6,6^{\mathrm{a}, \mathrm{b}}$ & $136,0 \pm 9,3^{b}$ & $130,4 \pm 9,9^{b}$ & $129,2 \pm 8,6^{b}$ & $128,3 \pm 9,9^{b}$ & $129,1 \pm 6,9^{b}$ & $129,8 \pm 6,8^{b}$ & $130,9 \pm 8,4^{\mathrm{b}}$ \\
\hline \multirow{3}{*}{$\begin{array}{c}\text { PAM } \\
(\mathbf{m m H g})\end{array}$} & $\mathbf{I}$ & $106,2 \pm 12,6^{a}$ & $78,3 \pm 12,4^{\mathrm{a}}$ & $78,7 \pm 11,4^{\mathrm{a}}$ & $67,9 \pm 8,7^{8}$ & $68,5 \pm 9,7^{\mathrm{a}}$ & $74,1 \pm 12,9^{\mathrm{a}}$ & $76,2 \pm 9,2^{\text {a }}$ & $84,1 \pm 10,2^{\text {a }}$ \\
\hline & II & $99,3 \pm 14,0^{a}$ & $84,3 \pm 14,8^{\mathrm{a}}$ & $84,5 \pm 18,2^{a}$ & $75,7 \pm 16,6^{\mathrm{ab}}$ & $75,6 \pm 9,6^{a . b}$ & $75,0 \pm 9,2^{\mathrm{a}, \mathrm{b}}$ & $77,3 \pm 10,5^{\mathrm{a}}$ & $82,7 \pm 14,2^{8}$ \\
\hline & III & $100,0 \pm 11,1^{a}$ & $98,4 \pm 16,4^{b}$ & $94,4 \pm 13,8^{a}$ & $84,7 \pm 10,1^{a}$ & $82,3 \pm 7,2^{\mathrm{a}}$ & $85,3 \pm 12,6^{a}$ & $93,0 \pm 10,8^{\circ}$ & $95,1 \pm 15,8^{\mathrm{a}}$ \\
\hline \multirow{3}{*}{$\begin{array}{r}\text { PAD } \\
(\mathbf{m m H g})\end{array}$} & $\mathbf{I}$ & $86,3 \pm 18,3^{a}$ & $54,7 \pm 12,5^{\mathrm{a}}$ & $55,0 \pm 10,4^{a}$ & $48,0 \pm 8,1^{\mathrm{a}}$ & $46,5 \pm 7,0^{\mathrm{a}}$ & $54,3 \pm 11,5^{\mathrm{a}}$ & $59,3 \pm 9,5^{a}$ & $66,3 \pm 9,5^{\text {a }}$ \\
\hline & II & $81,0 \pm 18,1^{a}$ & $66,7 \pm 17,2^{\mathrm{a}}$ & $63,7 \pm 20,4^{\mathrm{a}}$ & $59,0 \pm 19,8^{2}$ & $53,6 \pm 9,0^{a . b}$ & $51,8 \pm 7,0^{\mathrm{a}}$ & $61,3 \pm 14,2^{a}$ & $64,7 \pm 15,2^{\mathrm{a}}$ \\
\hline & III & $78,2 \pm 16,4^{a}$ & $83,3 \pm 16,3^{b}$ & $71,9 \pm 15,6^{a}$ & $61,4 \pm 8,8^{a}$ & $59,8 \pm 7,7^{\mathrm{b}}$ & $66,2 \pm 13,3^{b}$ & $68,8 \pm 8,4^{\mathrm{a}}$ & $78,3 \pm 10,4^{b}$ \\
\hline \multirow{3}{*}{$\begin{array}{c}\text { FR } \\
(\mathrm{mov} \text { resp/min) }\end{array}$} & $\mathbf{I}$ & $27,4 \pm 6.7^{a}$ & $28,3 \pm 17,7^{\mathrm{a}}$ & $25,4 \pm 13,9^{\mathrm{a}}$ & $38,6 \pm 28,8^{a . b}$ & $51,9 \pm 27,4^{a}$ & $33,3 \pm 12,6^{\mathrm{a}}$ & $26,1 \pm 14,1^{2}$ & $23,5 \pm 9,1^{\mathrm{a}}$ \\
\hline & II & $26,7 \pm 9,9^{x}$ & $25,8 \pm 16,3^{\mathrm{a}}$ & $15,8 \pm 7,8^{2}$ & $16,8 \pm 6,9^{\mathrm{a}}$ & $28,0 \pm 13,5^{\mathrm{a}}$ & $29,7 \pm 13,7^{\mathrm{a}}$ & $21,6 \pm 7,4^{\text {a }}$ & $20,2 \pm 5,6^{\mathrm{a}}$ \\
\hline & III & $28,6 \pm 9,3^{\mathrm{a}}$ & $63,8 \pm 55,7^{\circ}$ & $65,4 \pm 56,6^{b}$ & $70,1 \pm 49,9^{b}$ & $106,0 \pm 41,8^{b}$ & $95,8 \pm 53,0^{b}$ & $78,1 \pm 53,0^{b}$ & $44,9 \pm 41,8^{\mathrm{a}}$ \\
\hline \multirow[t]{3}{*}{ pH } & I & $7,4 \pm 0,04^{\text {a }}$ & $7,35 \pm 0,05$ & & $7,30 \pm 0,06^{a}$ & & $7,36 \pm 0,05^{a}$ & & $7,38 \pm 0,03^{2}$ \\
\hline & II & $7,4 \pm 0,03^{\mathrm{a}}$ & $7,42 \pm 0,03$ & & $7,34 \pm 0,05^{\mathrm{a}}$ & & $7,40 \pm 0,04^{\mathrm{a}}$ & & $7,40 \pm 0,05^{a}$ \\
\hline & III & $7,43 \pm 0,03^{n}$ & $7,37 \pm 0,03$ & & $7,29 \pm 0,03^{\mathrm{a}}$ & & $7,34 \pm 0,03^{b}$ & & $7,40 \pm 0,04^{a}$ \\
\hline \multirow{3}{*}{$\begin{array}{c}\mathrm{PaO}_{2} \\
(\mathrm{mmHg})\end{array}$} & $\mathbf{I}$ & $85,9 \pm 8,7^{a}$ & $79,0 \pm 12,3^{\mathrm{a}}$ & & $72,5 \pm 6,6^{\mathrm{a}}$ & & $83,7 \pm 7,9^{a}$ & & $89,4 \pm 9,5^{3}$ \\
\hline & II & $88,8 \pm 7,1^{\text {a }}$ & $87,4 \pm 8,6^{a}$ & & $74,4 \pm 12,2^{\mathrm{a}}$ & & $86,9 \pm 8,9^{a}$ & & $90,3 \pm 9,0^{\mathrm{a}}$ \\
\hline & III & $88,8 \pm 7,1^{\text {a }}$ & $87,4 \pm 8,6^{\mathrm{a}}$ & & $74,4 \pm 12,2^{\mathrm{a}}$ & & $86,9 \pm 8,9^{\star}$ & & $90,3 \pm 9,0^{8}$ \\
\hline \multirow{3}{*}{$\begin{array}{c}\mathrm{PaCO}_{2} \\
(\mathrm{mmHg})\end{array}$} & I & $32,2 \pm 4,6^{\mathrm{a}}$ & $34,5 \pm 4,3^{2}$ & & $39,9 \pm 3,8^{a b}$ & & $36,1 \pm 2,6^{a, b}$ & & $32,0 \pm 3,2^{a}$ \\
\hline & II & $30,4 \pm 2,7^{\mathrm{a}}$ & $30,9 \pm 3,7^{a}$ & & $36,6 \pm 3,4^{a}$ & & $32,8 \pm 3,6^{2}$ & & $31,2 \pm 3,9^{\mathrm{a}}$ \\
\hline & III & $31,4 \pm 3,5^{\mathrm{a}}$ & $34,2 \pm 7,0^{\mathrm{a}}$ & & $41,5 \pm 2,4^{b}$ & & $37,4 \pm 2,6^{b}$ & & $33,9 \pm 3,8^{\mathrm{a}}$ \\
\hline
\end{tabular}

MPA- medicação pré-anestésica; DP - desvio-padrão; a,b,c- valores com letras não coincidentes apresentam diferença estatisticamente significativa $(\mathrm{p}<0,05)$ entre si, na comparação das médias entre os grupos.

quando comparado com os valores controle nos animais do GI. Em GII, decorridos 10 e 20 minutos após a administração do propofol, os valores foram superiores quando comparados aos valores-controle, tendo sido as diferenças significativas. O GIII apresentou FR superior e estatisticamente significativa aos três minutos após MPA, e aos um, cinco, 10,20 e 30 minutos pós-propofol, quando comparado com os grupos I e II.

Houve diminuição nos valores do $\mathrm{pH}$ nos animais do GI até os cinco minutos pós-propofol, voltando a se elevar posteriormente. Os valores obtidos após a administração da acepromazina e alfentanil e aqueles obtidos aos cinco e 20 minutos pós-propofol foram inferiores em relação aos valores-controle $(p<0,05)$. O decréscimo nos valores obtidos aos cinco minutos pós-propofol nos animais do GII foi estatisticamente significativo em relação aos valores controle. Os valores obtidos nos animais do GIII até os 20 minutos pós-propofol mostraramse significativamente inferiores em relação aos valores controle $(\mathrm{p}<0,05)$ (Tabela 1$)$.

Em relação aos animais do GI, houve diminuição gradativa nos valores da $\mathrm{PaO}_{2}$ até os cinco minutos pós-propofol $(\mathrm{p}<0,05)$. Os valores obtidos no GII aos cinco minutos pós-propofol foram inferiores em relação aos demais momentos 
de observação( $p<0,05)$. No GIII, aos três minutos pós-alfentanil, observou-se queda na $\mathrm{PaO}_{2}$, que foi estatisticamente significativa em relação aos valorescontrole. Essa queda acentuou-se aos cinco minutos após a administração do propofol e os valores obtidos foram inferiores em relação aos valores prévios ao experimento (Tabela l).

Observou-se aumento nos valores da $\mathrm{PaCO}_{2}$ nos animais do GI e GIII aos cinco e 20 minutos pós-propofol em relação aos valores controle $(\mathrm{p}<0,05)$. Os valores obtidos aos cinco minutos após a administração do propofol no GII foram superiores em relação a todos os demais tempos de avaliação $(p<0,05)$. Os valores obtidos aos cinco e 20 minutos após a administração do propofol no GIII foram superiores quando comparados com os valores avaliados no GII $(p<0,05)$. Em relação ao bicarbonato plasmático, verificou-se ligeiro aumento nos animais do GII, observado aos cinco e 20 minutos após administração do propofol $(\mathrm{p}<0,05)$ (Tabela l).

Os valores dos níveis de $\mathrm{SaO}_{2}$ avaliados após administração da acepromazina e alfentanil foram inferiores aos valores-controle e aos valores obtidos ao término do experimento nos três grupos $(\mathrm{p}<0,05)$. Houve decréscimo gradativo na temperatura retal após a administração das drogas nos três grupos experimentais $(\mathrm{p}<0,05)$. O tempo de latência do propofol foi $26,4 \pm 4,1$ s GI, $28,1 \pm 3,8$ s e $28,8 \pm 4,5 \mathrm{~s}$ no GIII, não tendo sido observadas diferenças significativas entre os três grupos de experimentação. A realização da intubação orotraqueal foi extremamente fácil em todos os animais dos três grupos experimentais, sendo necessária apenas uma tentativa. Não ocorreram fenômenos excitatórios na indução anestésica em qualquer dos animais dos três grupos experimentais. A analgesia e o relaxamento muscular apresentados após a utilização do propofol e avaliados através de escore específico estão demonstrados na tabela 2 . Em relação à analgesia, apenas aos cinco minutos os grupos apresentaram escore com diferença estatisticamente significativa. Aos 10 e 30 minutos após a administração do propofol, os grupos apresentaram escores com diferenças estatisticamente significativa em relação ao miorrelaxamento. Em 20\% dos animais dos grupos I e II e $40 \%$ do grupo III, foi possível observar incoordenação motora durante $\mathrm{o}$ período de recuperação anestésica. O período hábil anestésico foi $17,6 \pm 4,74 \mathrm{~min}$ no GI, 15,0 $\pm 5,12 \mathrm{~min}$ no GII e $15,0 \pm 3,56 \mathrm{~min}$ no GIII, não tendo sido observadas diferenças significativas entre os três grupos.

\section{DISCUSSÃO}

Os animais do GIII apresentaram diminuição da frequência cardíaca em torno de $49 \%$, logo após a administração do alfentanil. O mesmo foi observado por ILKIW et al. (1991) ao compararem a farmacocinética do alfentanil em coelhos, ovinos e cães, e constataram o decréscimo médio da frequência cardíaca de $59 \%$ durante a infusão da droga. D'AUBIOUL et al. (1984), ao avaliarem o alfentanil em cães com doses crescentes, verificaram bradicardia significativa, alcançando o valor mínimo após a administração de $400 \mu \mathrm{g} / \mathrm{kg}$. Esses dados contrastam com os resultados de KEIN et al. (1986), que usaram $45 \mu \mathrm{g} / \mathrm{kg}$ de alfentanil na dose de indução seguida de $3 \mu \mathrm{g} / \mathrm{kg} /$ minuto em cães anestesiados com halotano, e não observaram alterações significativas na frequência cardíaca. Os animais que receberam a associação acepromazina/alfentanil também mostraram redução na frequência cardíaca, porém de menor intensidade. Essa diminuição da frequência cardíaca nos grupos I e III foi revertida após a administração do propofol. A bradicardia induzida pelo alfentanil é, provavelmente, decorrente dos mesmos mecanismos responsáveis pela bradicardia promovida pelo fentanil. Embora existam ainda algumas dúvidas concernentes aos mecanismos desse efeito, estudos experimentais sugerem que a bradicardia seja causada por estimulação do núcleo vagai (REITAN et al., 1978). Os animais do GII apresentaram taquicardia um minuto após a administração do propofol. Esse incremento também foi descrito por HALL \& CHAMBERS (1987), TAYLOR (1989), FONDA (1991) e VAINIO (1991) sendo que MANDSAGER $\boldsymbol{e} \boldsymbol{t} \boldsymbol{a l}$. (1991) e ROBERTSON $\boldsymbol{e} \boldsymbol{t} \boldsymbol{a l}$. (1991) observaram bradicardia após seu uso. GROUNDS et al. (1985) e MONK et al. (1987), ao avaliarem os efeitos do propofol em seres humanos, relataram que as alterações na frequência cardíaca foram variáveis e insignificantes. Sabe-se que o propofol pode apresentar efeitos vagotônico ou simpatolítico centrais (CULLEN et al., 1987). Alguns autores atestam que após o uso do propofol, existe diminuição da sensibilidade dos barorreceptores (CULLEN $\boldsymbol{e t}$ al., 1987; SAMAIN $\boldsymbol{e t}$ $\boldsymbol{a l}$. , 1987) às variações na pressão arterial. Neste experimento, pode-se constatar que o propofoi desencadeou taquicardia quando da presença de níveis pressóricos inferiores aos basais, igualmente aos resultados observados por HALL \& CHAMBERS (1987), TAYLOR (1989), e FONDA (1991).

$\mathrm{Na}$ análise da pressão arterial, pode-se observar diminuição nos componentes sistólico, médio e diastólico após a administração do propofol nos grupos I e II. Tal diminuição foi significativa em relação a todos os demais tempos de avaliação, com recuperação próxima aos valores controle ao término 
Tabela 2 - Avaliação percentual dos cães dos grupos I (acepromazina, alfentanil e propofol), II (acepromazina e propofol) e III (alfentanil e propofol) em relação à analgesia e relaxamento muscular (segundo escore proposto), nos diferentes tempos de avaliação após a administração do propofol

GRUPOS MINUTOS APÓS PROPOFOL

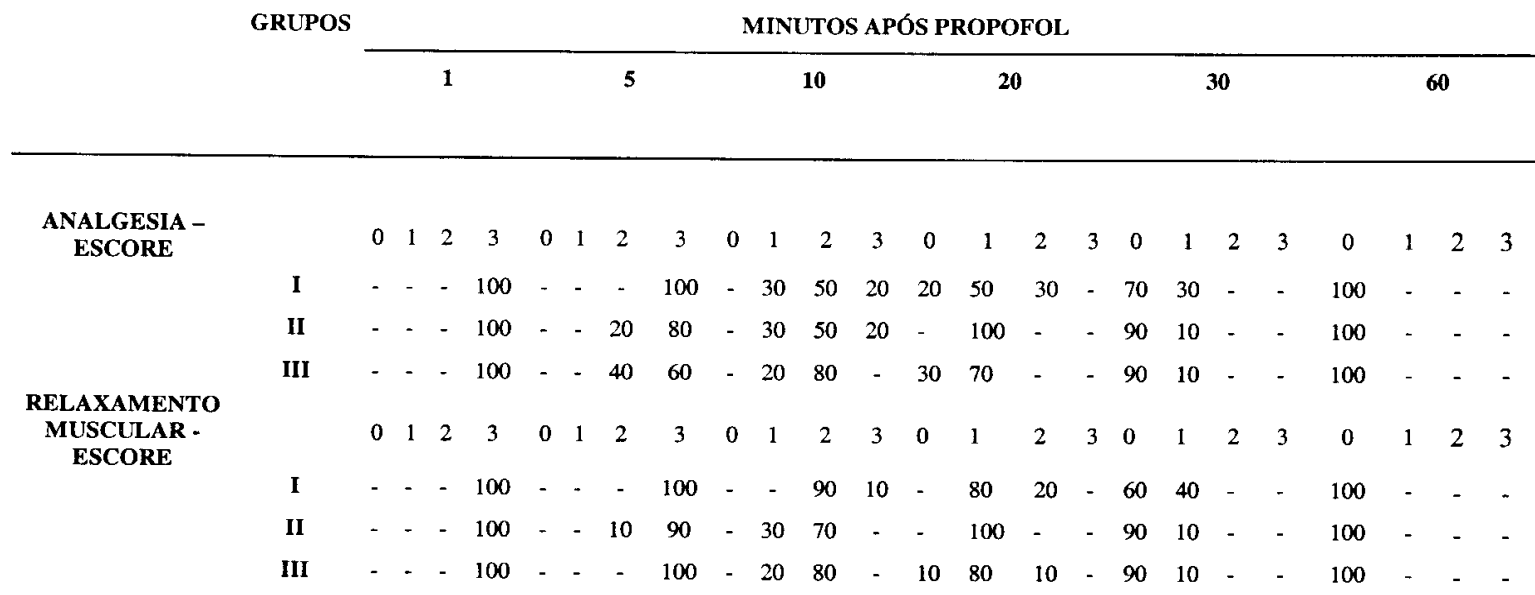

Analgesia: 0 = ruim; 1 = razoável; 2 = boa e 3 = excelente

Relaxamento Muscular: $0=$ ausente; 1 = leve; 2 = regular e $3=$ intenso

do experimento. Essa redução também foi constatada por HALL \& CHAMBERS (1987) e ILKIW et al. (1992) em cães, não tendo sido observada por FONDA (1991) que relatou aumento na pressão arterial após a infusão da droga em cães. Diferentes razões têm sido sugeridas para a diminuição na pressão arterial associada ao propofol. Muitos pesquisadores atestam a combinação da redução do débito cardíaco e da resistência vascular sistémica como sendo os responsáveis (GROUNDS et al., 1985; MONK et al., 1987; CLAEYS et al., 1988; BRUSSEL et al., 1989). O decréscimo observado na resistência vascular sistémica pode ser o resultado da depressão causada pela droga nos centros vasomotores localizados no tronco cerebral (MONK et al., 1987), ou efeito direto da roga no músculo liso vascular, ou por promoverem alteração na atividade barorreceptora (BRUSSEL et al., 1989). A diminuição mais acentuada nos grupos I e II pode também advir da ação da acepromazina, que provoca vasodilatação periférica, sendo bastante conhecida sua ação hipotensora (PUGH, 1964; COHEN \& TILLEY, 1979). A capacidade dos opióides em manter a estabilidade hemodinâmica é bem conhecida. ILKIW et al. (1991), ao compararem a farmacocinética do alfentanil em coelhos, ovinos e cães, constataram que o alfentanil não causou alteração significativa na pressão arterial média. NAUTA et al. (1983) compararam os agentes alfentanil, tiopental, midazolam e etomidato, e observaram estabilidade pressórica após a administração do alfentanil, tendo sido considerado superior em relação aos demais. Assim, a queda apenas da PAD e PAM, observada no grupo III, pode ser explicada pela redução da resistência vascular sistémica como aponta a literatura mencionada.

O cão pode apresentar bloqueio sino-atrial (BSA) sem a existência de cardiopatia. Normalmente, ocorre em cães braquicefálicos devido ao estímulo vagal durante a respiração ou simplesmente por aumento do tônus vagal (EDWARDS, 1987). O fentanil e os seus congéneres promovem bradicardia (REITAN $\boldsymbol{e t}$ al., 1978), o que explica a maior incidência de BSA que se verificou nos animais dos grupos I e III os quais receberam alfentanil. Já o propofol, que promoveu aumento da frequência cardíaca, suprimiu em 100\% os distúrbios do ritmo durante 20 minutos nos animais do grupo I e III. O propofol apresenta meia-vida de eliminação de 84 minutos sendo agente de curta duração (HASKINS, 1992). Devido a essa característica farmacocinética, pode-se entender a presença de BSA decorridos 20 minutos de sua administração, pois, a partir desse tempo, sua ação taquicardizante não estaria mais presente, havendo predominância da ação do alfentanil ou pelo fato desses distúrbios serem fisiológicos nestes animais. Os fenotiazínicos promovem freqüentemente graus variados de bloqueio átrio-ventricular (BAV) no cão e eventualmente BSA (MELLER \& TILLEY, 1988)

Ciência Rural, v. 30, n. 4, 2000. 
como pode-se comprovar eletrocardiografícamente nos animais do grupo II, que também foram suprimidos pela ação do propofol. Comparativamente porém, o alfentanil foi o responsável pelo aparecimento do maior número de BSA e demais distúrbios da condução, já que a maior incidência desses eventos ocorreu nos animais do grupo III (15\%) contra 8,7\% dos animais de cada um dos grupos I e II. D'AUBIOUL et al (1984) também observaram distúrbios da condução átrioventricular ao utilizarem o alfentanil em altas doses no cão. Já FLECKNELL et al. (1990) obtiveram assistolia atrial em $20 \%$ dos cães que foram submetidos à associação de alfentanil e propofoi. Essa assistolia foi convertida a bloqueio átrioventricular e eventualmente a ritmo sinusal após a administração da atropina.

Sabe-se que os opióides promovem depressão respiratória em graus variados, de acordo com a droga em questão e a dose administrada. A depressão respiratória se traduz por frequências respiratórias baixas, às vezes apnéia ou taquipnéia (De CASTRO et al., 1979). Em relação aos gases sanguíneos, observam-se aumento da $\mathrm{PaCO}_{2} ; \mathrm{e}$ diminuição da $\mathrm{PaO}_{2}$, podendo haver em decorrência disso, valores alterados do $\mathrm{pH}$ sanguíneo (D'AUBIOUL et al, 1984; ARNDT et al., 1986). O propofol, por sua vez, também promove depressão respiratória por deprimir diretamente o centro respiratório e a resposta ventilatória aos valores de $\mathrm{PaCO}_{2}$. Essa depressão é caracterizada por redução da frequência respiratória (GENEVOIS et al., 1988; CHAMBERS, 1989; FAU et al., 1989; MORGAN \& LEGGE, 1989; TAYLOR, 1989; ROBERTSON et al., 1991; HALL \& CLARKE, 1991; ILKIW et $\boldsymbol{a l}$., 1992) e do volume minuto (TAYLOR, 1989). Assim, pode-se observar a ação depressora do alfentanil no presente experimento, quando verificou-se que os animais do grupo I apresentaram taquipnéia exacerbada aos 10 minutos pós-propofol (que correspondem a 13 minutos da administração do alfentanil), hipoxemia e hipercapnia aos cinco e 20 minutos pós-propofoi e acidemia extremamente significativa aos cinco minutos. Essas observações no atinente à ação depressora do alfentanil foram corroboradas pela acentuada depressão respiratória notada nos animais tratados apenas com a associação de alfentanil e propofol. Verificaram-se aumento exacerbado da frequência respiratória em todos os momentos que seguiram a administração do alfentanil com redução do $\mathrm{pH}$, e aumento da $\mathrm{PaCO}_{2}$; a partir de sua administração, hipercapnia esta que foi acentuada após a aplicação do propofol. Também observou-se redução gradativa da $\mathrm{PaO}_{2}$ com depressão mais significativa após a administração do propofol, sendo que a $\mathrm{SaO}_{2}$ teve o mesmo comportamento. O alfentanil foi o fármaco que mais causou alterações na função respiratória, e isso pode ser bem salientado quando se observaram os resultados do GII, no qual as alterações dos gases sanguíneos e do $\mathrm{pH}$ somente foram verificadas cinco minutos após a administração do propofol. Isso toma também evidente a ação depressora do propofol na ventilação pulmonar, que certamente contribuiu bastante nas alterações verificadas nos grupos I e III. Esses resultados coincidem com os achados de WATKINS et al. (1987), MANDSAGER et al. (1991), VAINIO (1991) e ILKIW et al. (1992) que também obtiveram diminuição da $\mathrm{PaO}_{2}$, aumento da $\mathrm{PaC} 02$ e diminuição do $\mathrm{pH}$, três a cinco minutos após a administração do propofol, refletindo ligeira acidose respiratória. Constataram-se episódios de apnéia após a administração de propofol em 10\% dos animais de cada grupo experimental, sendo que o período de apnéia variou entre 15 e 30 segundos. MORGAN \& LEGGE (1989) notaram que a apnéia foi o efeito mais comum durante a indução com propofol. GILLIES \& LEES (1989) relacionaram esse efeito depressor à velocidade de administração do fármaco; pacientes que receberam o propofol por 80 segundos tiveram baixa incidência de apnéias prolongadas (10\%) quando comparados com aqueles que receberam o agente durante 20 segundos (40\%). SMITH et al. (1993) obtiveram apnéia em 85\% dos cães estudados associada ao incremento na $\mathrm{PvC0} 2$ e resultante diminuição no $\mathrm{pH}$. Há relatos dos efeitos depressores também do alfentanil. CHAMBERS (1989), ao utilizar dose baixa de alfentanil $(10 \mu \mathrm{g} / \mathrm{kg})$, obteve apnéia em $11 \%$ dos animais por mais de três minutos de duração. TAYLOR $\boldsymbol{e t} \boldsymbol{a l}$. (1986), ao avaliarem o propofoi e tiopental com diferentes pré-medicações, constataram que a ocorrência de apnéias foi maior no grupo que recebeu opióides previamente. Isso contraria nossos resultados, pois os grupos I e III, que receberam alfentanil, tiveram a mesma incidência de apnéias que o grupo que recebeu apenas acepromazina.

A diminuição significativa observada na temperatura retal nos três grupos experimentais foi também descrita por FONDA (1991) e seria atribuída a menor atividade do centro termorregulador hipotalâmico (HALL \& CLARKE, 1991) produzida pela maioria dos agentes anestésicos. Em relação aos grupos I e II, pode-se também relacionar essa queda da temperatura à perda de calor que os fenotiazínicos provocam devido à vasodilatação periférica produzida (HALL \& CLARKE, 1991). Os opióides agonistas de receptores $m u$ podem causar redução da temperatura corpórea (HASKINS, 1992), o que contribuiria também para a diminuição desse parâmetro nos grupos I e III. 
Os animais dos grupos I e II que receberam inicialmente acepromazina apresentaram decúbito espontâneo em poucos minutos, com diminuição da resposta aos estímulos audiovisuais, presença de ptose palpebral e protrusão da terceira pálpebra, características do efeito tranquiilizante promovido por esse fármaco (PUGH, 1964). No grupo I, após a aplicação do alfentanil, os animais continuaram em decúbito lateral e apenas um deles se apresentou com respiração ofegante, durante dois a três minutos seguintes. Apesar da tendência dos opióides promoverem depressão respiratória, algumas vezes esses fármacos induzem um efeito no centro termorregulador, o qual, equivocadamente, interpreta a temperatura corpórea como sendo elevada, ocorrendo assim a alteração no padrão respiratório (McKEL-VEY \& HOLLINGSHEAD 1994). Os animais do grupo III apresentaram decúbito lateral em poucos segundos após à administração do alfentanil. Em 50\% deles, pode-se observar a ocorrência de delírio nos 15 a 20 segundos subsequentes a administração. Tal delírio perdurou por cerca de um a dois minutos e foi caracterizado por vocalização, olhar fixo e movimentos de pedalagem. ARNDT et al. (1986), ao estudarem doses crescentes do alfentanil (10, 20, 80,160 e $320 \mu \mathrm{g} / \mathrm{kg}$ ) em cães não anestesiados, não relataram esse comportamento; apenas descreveram sedação após a quarta dose. DOHOO et al. (1986), ao compararem a sedação da associação oximorfona/acepromazina com a associação fentanil/droperidol em cães, observaram como efeitos indesejáveis mais freqüentes, a respiração ofegante e a alteração na resposta aos estímulos auditivos. Conforme relatado por BEDNARSKI (1992), a administração prévia de tranqüilizante diminuiu a incidência de excitação produzida pêlos opióides no presente trabalho. Pode-se verificar, neste estudo, a ocorrência de defecação em $20 \%$ dos animais de cada um dos grupos I e III após a administração do alfentanil, que também foi descrita por DOHOO et al. (1986). Isso decorre dos efeitos estimulantes dos opióides no núcleo vagal (REITAN et al., 1978) que desencadeiam aumento na atividade gastrintestinal (HALL \& CLARKE, 1991). Essa ação também justificaria a salivação profusa ocorrida em $20 \%$ dos animais do grupo III, segundos após a administração do alfentanil. A descrição da atividade antisialogoga dos fenotiazínicos por MASSONE (1999) explicaria a ausência da salivação no grupo I, no qual os animais receberam acepromazina e alfentanil. A administração do propofol durante o período de 20 segundos foi convencionada na tentativa de avaliar-se melhor o tempo de latência do fármaco nos diferentes grupos experimentais e as possíveis alterações decorrentes dessa velocidade instituída. GEEL (1991) observou que a velocidade de administração do propofol afetou a dose requerida para intubação orotraqueal e o tempo de latência da droga, pois quando administrado lentamente (durante aproximadamente 60 segundos), necessitou de maiores doses do agente para efetuar a intubação orotraqueal. GENEVOIS $\boldsymbol{e t}$ al. (1988) não relacionaram o tempo de administração a qualquer alteração de dose ou latência, ao terem administrado o propofoi em período que variou de oito a 73 segundos, no qual obtiveram período de latência médio de 18,7 segundos.

Apesar das características físicas (emulsão de óleo em água), a administração do propofol foi facilmente realizada através do catéter inserido. Dor à injeção, freqüente achado no homem após a administração do propofol (MACKENZIE \& GRANDT, 1985), não foi evidenciada neste experimento, o mesmo relatado por WATKINS et al. (1987) e MORGAN \& LEGGE (1989). WEAVER \& RAPTOPOULOS (1990), ao terem utilizado propofol na indução anestésica de cães e gatos, observaram resposta à dor em apenas um cão. A ausência aparente, ou a baixa incidência de dor à injeção do propofol em cães, parece estar relacionada ao uso de veia calibrosa e às dificuldades em avaliar algumas reações subjetivas dos animais durante sua administração (FLECKNELL $\boldsymbol{e t}$ al., 1990). Nenhum dos animais apresentou excitação durante a aplicação do propofol, tendo sido a indução suave e tranqüila, fato bem ressaltado na literatura nas diferentes espécies animais (GLEN, 1980; WATKINS et al,1987; GENEVOIS et al., 1988; FAU et al., 1989; MORGAN\& LEGGE, 1989; FLECKNELL $\boldsymbol{e}$ t al., 1990; WEAVER \& RAPTOPOULOS, 1990; SMITH et al., 1992; VAINIO, 1991; QUANDT \& ROBINSON, 1992). O mesmo tem sido descrito na literatura humana (SEBEL \& LOWDON, 1989), onde o tempo curto de indução sem efeitos excitatórios toma esse fármaco comparável de modo superior a outros agentes. WATNEY \& PABLO (1992) não observaram sinais de excitação na indução de cães pré-tratados com acepromazina; em contraste, $14 \%$ dos animais não pré-tratados apresentaram fenômenos excitatórios, sendo que os autores relacionaram tais ocorrências ao plano superficial de anestesia. MANDSAGER et $\boldsymbol{a l}$. (1991) obtiveram melhor qualidade de indução anestésica em cães com propofol, quando compararam com a associação acepromazina/diazepam/quetamina. No presente estudo, pode-se notar a ocorrência de mioclonias após o uso do propofol em dois cães do grupo I (6,66\% de todos os animais deste experimento) fato que também foi relatado por HALL \& CHAMBERS (1987), WATKINS et al. (1987), CHAMBERS 
(1989) e DAVIES (1991). HALL \& CHAMBERS (1987), ao avaliarem a manutenção anestésica com propofol em cães, constataram ainda tremores dos membros, tosse, e alta incidência de vômito no período de recuperação. DAVIES (1991) estudou os fenômenos excitatórios após o uso do propofol em cães. Ao avaliar 159 anestesias, observou a ocorrência de tais fenômenos em 7,5\% delas, e pode constatar, além das mioclonias, movimentos de pedalagem, opistótono e rigidez de membros. Esses efeitos excitatórios podem ser devido à ação específica do agente no sistema nervoso central que desencadearia atividade semelhante às convulsões (vias dopaminérgicas) (HOPKINS, 1988).

A analgesia considerada adequada (excelente ou boa) de acordo com o escore proposto, só foi encontrada durante 10 minutos nos três grupos. Assim, em relação ao método de avaliação utilizado, pode-se verificar que as diferentes medicações pré-anestésicas não intensificaram a analgesia, uma vez que o tempo médio de hiporresponsividade não apresentou diferença significativa entre os grupos. De acordo com a literatura consultada, pode-se observar que o método de avaliação proposto é indicado (GENEVOIS et al., 1988; VAINIO, 1991), porém, tal verificação é melhor efetuada através de procedimentos cirúrgicos (WATKINS et al., 1987; FAU et al., 1989). Em relação à completa recuperação, os resultados obtidos suportam os achados de outros autores nos quais a recuperação foi rápida e isenta de fenómenos excitatórios (WATKINS et al., 1987; FAU et al; 1989; GENEVOIS et al., 1988; MORGAN \& LEGGE, 1989; WEAVER \& RAPTOPOULOS, 1990;ROBERTSON et al, 1991; VAINIO, 1991).

Os resultados obtidos permitem concluir que a indução da anestesia promovida pelo propofol é suave e tranqüila, e que a acepromazina e a associação de acepromazina e alfentanil são medicações pré-anestésicas efetivas pois contribuem para a obtenção de técnica anestésica isenta de efeitos adversos. O uso de alfentanil promove bradiarritmias, depressão respiratória, assim como delírios, alterações que contra-indicam sua utilização isolada.

\section{REFERÊNCIAS BIBLIOGRÁFICAS}

ARNDT, J.O., BEDNARSKI, B., PARASHER, C. Alfentanil's analgesic, respiratory and cardiovascular actions in relation to dose and plasma concentration in unanesthetized dogs. Anesthesiology, v.64, p.345-352, 1986

BEDNARSKI, R.M. Precautions when using opioid agonists for inducdon of anesthesia. The Veterinary Clinics of North America - Small Animal Practice, v.22, n.2, p. 273-275, 1992.
BRUSSEL, T., THEISSEN, J.T., VIGFUSSON, G., et al Hemodynamic and cardiodynanüc effects of propofol and etomidate: negative inotropic properties of propofol. Anesthesia and Analgesia, v.69, p.35-40,1989.

CHAMBERS, J.P. propofol infusion anaesthesia in dogs. Joumal of the Association of the Veterinary Anaesthetits, v. $15, \mathrm{p} .135,1988$.

CHAMBERS, J.P. Induction of anaesthesia in dogs with alfentanil and propofol. Journal of the Association of the Veterinary Anaesthetists, v.16, p.14-17,1989.

CLAEYS, M.A., GEPTS, E., CAMU, F. Haemodynamic changes during anaesthesia induced and maintained with propofol. British Journal of Anaesthesia, v.600, p.3-9, 1988.

COHEN, R.B., TILLEY, L.P. Cardiac arrytmias in the anesthetized patient Veterinary Clinics of North América - . Small Animal Practice, v.9, n.1, p.155-167, 1979.

CULLEN, P.M., TURTLE, M., PRYS-ROBERTS, C., et al Effect of propofol anesthesia on baroreflex activity in humans. Anesthesia and Analgesia, v.66, p.1115-120,1987.

D'AUBIOUL, J., VAN GERVEN, W., VAN DE WATER, A., $\boldsymbol{e}$ al Cardiovascular and some respiratory effects of high doses of alfentanil in dogs. European Journal of Pharmacology, v.100,p.79-84,1984.

DAVIES, C. Excitatory phenomena following the use of propofol in dogs. Journal of Veterinary Anaesthesia, v. 18, p.4851,1991 .

DeCASTRO, J., VAN DE WATER, A., WOUTERS, L., et al Comparative study of cardiovascular, neurological and metabolic side effects of eight narcotic in dogs. Acta Anaesthesiology Bélgica, v.30, p.5-99,1979.

DOHOO, S.E., O'CONNOR, M.K., McDONELL, W.N., et al. A clinical comparison of oxymorphone/acepromazine and fentanyl/droperidol sedation in dogs. Journal of the American Animal Hospital Association, v.22, p.313-317, 1986.

EDWARDS, N.J. Bolton's handbook of canine and feline eletrocardiography. 2 ed. Philadelphia : Saunders, 1987. p.60-84.

PAU, D., GUYONNET, V., GENEVOIS, J.P. et al Utílisation chez lê chien d'un novel anesthésique: lê propofol. Revue de Médecine Vétérinaire, v.140, n.10, p.891-898,1989.

FLECKNELL, P.A., KIRK, A.J.B., FOX, C.E., et al Long-term anesthesia with propofol and alfentanil in the dog and its partial reversal with nalbuphine. Journal of the Association of the Veterinary Anaesthetits, v.17, p.11-16,1990.

FONDA, D. Continuous infusion anesthesia with propofoi in dogs: clinically optimized dosages. In: INTERNATIONAL CONGRESS OF VETERINARY ANAESTHESIA, 4, Utrecht, 1991. Proceedings... Utrecht : State University of Utrecht, 1991. p.159-161.

GEEL, J.R. The effect of premedieation on the induction dose of propofoi in dogs and cats. Journal of the South African Veterinary Association, v.62, n.3, p.1 18-123, 1991.

GENEVOIS, J.P., FAU, D., FIENI, F., et al Utilization chez lê chien d'un novel anesthésique: lê propofol. 1: résultats après injection d'une dose unique de produit. Revue de Médecine Vétérinaire, v.139, n.12, p.1 119-1123,1988. 
GILLIES, G.W.A., LEES, N.W. on induction with propofol. 1989. The effects of speed of injecdon Anaesthesia, v.44, p.386-388,

LEN, J.B. Animal studies of the anaesthetic activity of ICI 35868 British Journal of Anaesthesia, v.52, p.731-742, 1980

GROUNDS, R.M., TWIGLEY, A.J., CARLI, F., et al. The haemodynamic effects of intravenous induction: comparison of the effects of Ihiopentone and propofol. Anaesthesia, v.40, p.735-740,1985.

HALL, L. W., CHAMBERS, J.P. A clinical trial of propofol infusion anaesthesia in dogs. Journal of Small Animal Practice, v.28, p.623-637,1987.

HALL, L. W., CLARKE, K.W. Veterinary anesthesia. 9 ed London: Baillière Tindall, 1991. 422p.

HASKINS, S.C. Injectable anesthetics. The Veterinary Clinics of North America - Small Animal Practice, v.22, n.2, p.245260,1992

HOPKINS, C.S. Recurrent opisthotonus associated with anaesthesia. Anaesthesia, v.43, p.904,1988

ILKIW, J.E., BENTHUYSEN, J.A., McNEAL, D. Comparative study of the pharmacokinetics of alfentanil in rabbits, sheep, and dogs. American Journal of Veterinary Research, v.52, n.4,p.581-584, 1991

ILKIW, J.E., PASCOE, P.J., HASKINS, S.C., et al Cardiovascular and respiratory effects of propofol administration in hypovolemic dogs. American Journal of Veterinary Research, v.53, n.12, p.2323-2327, 1992.

KEIN, N.D., REITAN, J.A., WHITE, D.A., et al Hemodynamic responses to alfentanil in halothane-anesthetized dogs. Anesthesia and Analgesia, v.65, p.765-770,1986.

MACKENZIE, N, GRANDT, I.S. Comparison of propofol with methohexitone in the provision of anesthesia for surgery under regional blockade. British Journal of Anaesthesia, v.57,p.1 167-1172,1985.

MANDSAGER, R.E., SHAWLEY, R.V., CLARK, D.M. Evaluation of propofol or acepromazine/diazepam/ketamine in the greyhound. Veterinary Surgery, v.20, n.1, p.80-81, 1991.

MASSONE, F. Anestesiologia veterinária. 3 ed. Rio de Janeiro Guanabara Koogan, 1999. Medicação pré-anestésica:p.17-30.

McKELVEY, D., HOLLINGSHEAD, K.W. Small animal anesthesia. St. Louis : Mosby, 1994. The preanesthetic period: p. $1-54$

MILLER, M.S., TILLEY, L.P. Eletrocardiography. In: FOX, R.P Canine and feline cardiology. New York : Churchill Uvingstone, 1988. p.43-89.

MONK, C.R., COATES, D.P., PRYS-ROBERTS, C., et al. Haemodynamic effects of a prolonged infusion of propofol as suppiement to nitrous oxide anaesthesia. British Journal of Anaesthesia, v.59, p.954-960,1987.

MORGAN, D.W.T., LEGGE, K. Clinical evaluation of propofol as an intravenous anaesthetic agent in cats and dogs. The Veterinary Record, v. 124, p.31-33,1989.

NAUTA, J, STANLEY, T.H., DE LANGE, S., et al. Anaesthetic induction with alfentanil: comparison with tiopental, midazolam, and etomidate. Canadian Anaesthetist's Society Journal, v.30, p.53-60,1983.

PUGH, D.M. Acepromazine in veterinary use. The Veterinary Record, v.76, n.16, p.439-443,1964.

QUANDT, J.E., ROBINSON, E.P. Propofol and thiopental: comparison of anesthetic effects in the dog. Veterinary Surgery, v.21, n.1, p.83,1992.

REITAN, J.A., STANGERT, K.B., WYMORE, M.L., $\boldsymbol{e} \boldsymbol{t} \boldsymbol{a l}$. Central vagal control of fentanyl induced bradycardia during halothane anesthesia. Anesthesia and Analgesia, v.57, p.3136,1978 .

ROBERTSON, S.A., JOHNSTON, S., BEEMSTERBOER, J. A comparison of propofol infusion anesthesia in greyhound and non-greyhound dogs. Veterinary Surgery, v.20, n.2, p.159, 1991.

SAMAIN, E., MARTY, J., GAUZIT, R., et al Effects of propofol on baroreflex control of heart rate on sympathetic activity in humans. Anesthesiology, v.67, A75,1987.

SANDERSON, J.H. Multicentre study of propofol in day case surgery. Anaesthesia, v.43, p.61-64, 1988.

SAWYER, D.C., BROWN, M., STRILER, E.L., et al Comparison of direct and indirect blood pressure measurement in anesmetized dogs. Laboratory Animal Science, v.41, n.2, p.134-138,1991.

SEBEL, P.S., LOWDON, J.D. Propofol: a new intravenous anesthetic. Anesthesiology, v.71, p.260-277, 1989.

SMITH, J., GAYNOR, J, BEDNARSKI, R. Clinical effects associated with propofol in dogs. Veterinary Surgery, v.21, n.1, p.84, 1992.

SMITH, J.A., GAYNOR, J.S., BEDNARSKI, R.M. et al Adverse effects of administration of propofol with various preanesthetic regimens in dogs. Journal of the America Veterinary Medical Association, v.202, p.1111-1115,1993.

TAYLOR, M.B., GROUNDS, R.M., MULROONEY, P.D., et al. Ventilatory effects of propofol during induction of anaesthesia: comparison with thiopentone. Anaesthesia, .41, p.816-820, 1986 .

TAYLOR, P.M. Adrenocortical response to propofol infusion in ponies: a preliminary report. Journal of the Association of the Veterinary Anaesthetists. v.16, p.12-14,1989.

TERZI, R.G.G. Equilíbrio ácido-básico e transporte de oxigénio. São Paulo : Manole, 1992. 267p.

VAINIO, O. Propofol infusion anaesthesia in dogs pre-medicated with medetornidine. Journal of Veterinary Anaesthesia, v.18,p.35-37,1991.

WATKINS, S.B., HALL, L.W., CLARKE, K.W. Propofol as na intravenous anesthetic agent in dogs. The Veterinary Record, v.120, n.14, p.326-329, 1987.

WATNEY, G.C.G., PABLO, L.S. Median effective dosage of propofol for induction of anesthesia in dogs. American Journal of Veterinary Research, v.53, n.12, p.2320-2327, 1992

WEAVER, B.M.Q., RAPTOPOULOS, D. Induction of anaesthesia in dogs and cats with propofol. The Veterinary Record, v.126, p.617-620,1990. 